\title{
Outcome measures for routine use in dementia services: some practical considerations
}

\author{
I J Higginson, P M Jefferys, C S Hodgson
}

\begin{abstract}
Objectives-To work with specialist community teams to assess the practicality and acceptability of identified outcome measures for routine use in dementia services.
\end{abstract}

Setting-Seven specialist dementia services: four multidisciplinary teams, a specialist service for carers, a community psychiatric nurse team, and a day hospital.

Subjects-20 members of staff from the specialist dementia services including psychiatry, community psychiatric nursing, social work, occupational therapy, Admiral nursing, ward management, geriatric nursing.

Main measure-A questionnaire designed to assess staff views on the use of six outcome measures in routine practice in terms of practicality, relevance, acceptability, and use in improving care.

Results-Each of the outcome measures took 15 to 30 minutes to administer. All were rated as easy to use and as relevant to dementia services and to carers. Staff commented that the measures could be useful in routine practice for structured assessment and service evaluation, but highlighted the need for sensitive use of measures with carers.

Conclusions-These measures consider the main domains of functioning for people with dementia and their carers. The measures are suitable for use in routine practice in dementia services and are acceptable to staff and carers. The project underlined the need for management support, staff ownership of measures, and training in using outcome measures. Staff concerns about service evaluation need to be acknowledged.

(Quality in Health Care 1997;6:120-124)

Keywords: outcome measures; routine practice; dementia

Harrow and Hillingdon Healthcare NHS Trust (Northwick Park Hospital), London

P M Jefferys, consultant psychiatrist on old age

Correspondence to: Professor I J Higginson, St Christopher's Hospice, 51-59 Lawrie Park Road, Sydenham, London SE26 $6 \mathrm{DZ}$.

Accepted for publication 5 June 1997 ure which considers all these factors and is feasible for routine use is not available. ${ }^{2} \mathrm{~A}$ further consideration in the evaluation of dementia services is that professional care usually includes both the person with dementia and their informal carer-for example, spouse, relative, friend. Most people with dementia are cared for in the community by an informal carer and rely on a healthy carer for their main source of support. Caring for someone with dementia can be physically and psychologically stressful. ${ }^{3}$ Therefore, outcome measures which reflect the aims of dementia services need to consider the health of both the person with dementia and their main carer.

This paper describes the first stages of a research project which aims to identify outcome measures from the medical literature and pilot their use in routine clinical practice with various specialist dementia services. An earlier systematic review ${ }^{2}$ identified potential measures from the medical literature according to criteria of validity, reliability, responsiveness to change, appropriateness of format, and length of administration. The review identified a total of 81 scales; 58 were relevant to the patient and 23 relevant to the informal carer.

Outcomes need to reflect the aims of the service and the needs and expectations of service users. The key objectives for dementia services were identified by questionnaires completed by general practitioners, district nurses, and specialist service professionals and by interviews with carers and excarers. ${ }^{4}$ Candidate measures from the literature review were then assessed against these objectives. The final shortlist included nine measures which met the literature criteria and service objectives.

Outcome measures will only be incorporated into practice if clinicians see their value and want to use them, and they will only be used properly if they understand the basics of methodology, have been trained to use them properly, and have the time to do so. ${ }^{5}$ Evaluations of outcome measures have not always been related to clinical experience and day to day practicalities and it is these factors which are likely to effect the probability of whether measures are accepted and used by service providers. This paper describes a practical approach to overcoming this by examining the shortlisted measures from our review which have already been proved to be valid and reliable, and testing them for practicality and acceptability in routine practice. Staff views of the perceived value of the measures is also considered, as is the effect of using outcome measures with carers. We hope that the details of the 
Table 1 Measures selected for testing

\begin{tabular}{|c|c|c|}
\hline Measure & Domains & Informant \\
\hline \multicolumn{3}{|l|}{ Patient measures: } \\
\hline Cornell scale for depression in dementia ${ }^{67}$ & Psychological, behaviour & Clinician interview with carer and client \\
\hline Alzheimer's deficit scale ${ }^{8}$ & Self care, behaviour, activities of daily living, social, cognitive & Clinician interview with carer \\
\hline Behaviour and mood disturbance scale ${ }^{9}$ & Psychological, behaviour & Carer completed \\
\hline \multicolumn{3}{|l|}{ Carer measures: } \\
\hline Perceived stress scale ${ }^{10-12}$ & Psychological & Carer completed \\
\hline Caregiver appraisal ${ }^{13}$ & Social, physical, psychological, knowledge & Carer completed \\
\hline \multicolumn{3}{|l|}{ Patient and carer measure: } \\
\hline Gilleard's problem checklist ${ }^{14}$ is $^{15}$ & $\begin{array}{l}\text { Patient: behaviour, activities of daily living, self care } \\
\text { Carer: social, psychological, physical }\end{array}$ & Carer completed \\
\hline
\end{tabular}

developmental methods, which are rarely described, will assist others introducing outcome measures for routine use.

\section{Method}

SETTINGS

Seven specialist dementia services were selected to reflect the range of community care available to people with dementia and their informal carers in 1996, and to include both inner city and suburban locations. The services comprised: four multidisciplinary elderly mental health teams (inner London), a specialist nursing service for dementia carers (London), a community psychiatric nurse team (east Hertfordshire) and a day hospital for older people (Harlow).

\section{PROCEDURE}

Box 1 shows the main elements of the outcome measures assessment phase.

- Shortlisting outcome measures:

Literature review of measures: reliable, valid, sensitive to change

Interview service providers and carers: service objectives

- Management support:

Present measures to managers and team leaders

- Staff ownership of measures: Teams select measures for piloting

- Staff training:

Training and practice in using outcome measures

- Staff support:

Clear guidelines and telephone helpline for staff

- Staff assessment of measures:

Staff use measures in practice and assess using feedback questionnaire

- Feedback to staff

Box 1 Key stages of the study to assess outcomes.

Management support

The research officer initially attended individual meetings with staff at management level from each of the services-for example, service managers, team leaders. These meetings aimed to gain management support for the project in terms of staff time and motivation, and a commitment to introducing outcome measures into routine practice.

Staff selection of measures

The project was presented to each clinical team at the weekly team meeting. The relevance and application of outcome measures in terms of quality of care was presented and discussed. Each member of staff was given a personal report which included a summary of the project and literature review and details of each of the nine shortlisted measures including background on their development and administration. Teams discussed each of the measures according to the following criteria:

- Validity of measure in relation to specific service aims

- Appropriateness of informant (patient/ clinician/carer)

- Appropriateness of format/administration for older people

- Appropriateness for use at all stages of dementia

- Ease of scoring and interpretation

- Wider applications - for example, to facilitate assessment

- Training requirements.

Three of the nine measures were discarded by teams, based on an unacceptable length of administration (too long for routine use), a need for specially trained raters, or unsuitable for use with people with severe dementia. The table illustrates the six measures finally selected by teams for piloting from the initial shortlist.

\section{Training in the use of outcome measures}

Individual staff varied greatly in their training needs and experience of using measures, with some staff having never been involved in any audit projects. To enhance the reliability and understanding of the measures, staff training in outcome measurement was provided. The most comprehensive training workshop offered included the role of outcome measures in improving care; training in administration, scoring and interpretation of the measures; experience of completing a measure; issues about using carers as informants; and data protection issues. Concerns about evaluation and confidentiality were often raised by staff and included concerns that data may be used to evaluate individual staff members or that the use of outcome measures could result in performance related pay. These issues, which are often major barriers to outcome measure use, were considered in detail.

\section{Staff support}

Step by step guidelines for assessing the measures were provided for each member of staff. These guidelines included a clear methodology for using the measures and assessing their use, as well as common questions which may arise-for example, what if the patient does not 
have an informal carer to act as proxy informant? What if the measure is only partially completed? What if I feel the carer is too distressed to act as respondent? A helpline was set up for staff to consider any queries throughout the study period.

\section{Staff assessment of the measures for use in routine practice}

Staff piloted one measure at a time with those patients and carers already known to the service, and who they thought would be willing and able to complete a measure. Each time a measure was piloted, staff completed a feedback questionnaire (appendix). This questionnaire assessed the practicality-for example, ease of administration, time taken to completeacceptability-for example, of format and the relevance of measures to carers - and use of the measures - for example, how useful the measures would be in routine practice, how useful was the data-with response options and space for additional comments. The results of the feedback questionnaire were presented in the form of simple descriptive statistics for the categorised items and illustrative comments from staff.

\section{Results}

Twenty staff were involved in piloting the six measures and staff reflected the multidisciplinary make up of teams including community psychiatric nurses, consultant psychiatrists, junior psychiatrists, social workers, occupational therapists, senior charge nurses, ward managers, and admiral nurses (specialist nurses for carers of people with dementia). A total of 45 completed feedback questionnaires were completed. Although all six measures were used within each service, most individual members of staff used two or three of the measures, depending on their case load and visits over the study period.

\section{PRACTICALITY}

All measures took less than 30 minutes to complete and on most occasions took less than 15 minutes. This variation did not depend so much on the individual measures as individual staff and situations. For example, a carer rated measure may take a while to administer if the carer takes the opportunity to discuss the situation relating to each item on the measure. When staff were asked, "How easy was the measure to use?" all measures were rated as quite easy or very easy to use and none as being very difficult to use. Asked, "Did you or the carer encounter any problems with completing the measure?", on 28 (70\%) occasions staff indicated that there had been no problems. Problems reported by staff were related to measure design such as the repetitive nature of some items, and the use of visual analogue scales with a carer group who may be unfamiliar with such rating methods and who tended to mark the extremes of the scale only, ignoring the continuum.
RELEVANCE

All measures were thought to be relevant (quite or very) to aspects of the specialist dementia service although no single measure encompassed all the relevant dimensions of functioning for both the patient and the carer. Carers in particular thought that the measures were relevant to their situation and staff comments indicated that carers identified with the items of the measures and this made them think that they were not the only ones to experience such problems. An important related issue highlighted by staff is that the process of completing the measures can also be emotive for some carers.

\section{USEFULNESS}

Staff were asked if the measures provided useful information and would be suitable to use in routine practice. On $84 \%$ of occasions respondents commented that the information was quite useful or very useful and none of the measures were rated as not at all useful. Staff commented that the measures were useful for detailed initial assessment. In terms of service quality, staff saw the value of using the outcome measures to assess change in functioning over time and to evaluate interventions. The measures also enabled discussion to take place on sensitive subjects which carers may have found difficult to bring up. Box 2 shows some examples of comments representing staff views.

\section{- Carer acceptability}

"The carer found it useful, actually spending time thinking about and realising some of the actions of her husbandshe took it for granted that these things occurred"

"(The carer) found the process of doing the exercise a little upsetting, realising just how poorly her husband is"

- Methodological points

"Some of the questions sound similar"

"Needed help with the scales ... tended just to mark extremes"

- Measuring change

"It would identify problems and changes so that care could be altered when the deficit score changed"

"As an assessment carried out at specific times to distinguish change and in what areas the change has occurred"

Box 2 Staff comments.

\section{Discussion}

One of the most common obstacles to using outcome measures in routine practice voiced by clinicians has been the practical aspect of a lack of time. ${ }^{16}$ Length of administration was one of the criteria for shortlisting of measures. Administration of the measures selected in this study was $15-30$ minutes, which is generally clinically acceptable. The measures were selected for their simplicity and most clinicians did not experience any administration problems. Many of the staff involved had not previously administered these measures and it is 
likely that with practice there would be fewer problems.

The measures were shortlisted for use with dementia services, and all measures were rated as having relevance to the patient's or carer's situation. Staff also said that carers thought the measures to be relevant to their situation and some found the process helpful and enlightening. Many staff considered that these outcome measures could be used in routine practice to assess change over time. As an adjunct, the measures could be used to facilitate conversation with carers on sensitive subjects or those which carers may have difficulty in acknowledging. An important point was made by staff concerning the wording of instructions for carer completed measures. Staff drew attention to the possibility when using a problem checklist of raising carer concern about future problems. Instructions with carers therefore need to be sensitively worded.

This study has identified outcome measures which could be used in routine practice. All received positive feedback from staff. Each of the measures piloted provides data relating to particular aspects of dementia. However, during the feedback discussions it became clear that no single measure encompassed all the relevant domains of functioning for clients and carers that were highlighted as being important in our earlier review. An outcome measure suitable for routine use will have limited detail by comparison with research measures, by nature of the practical constraints.

It was not within the scope of this pilot study to analyse the data obtained from the outcome measures and assess their ability to influence care on a long term basis. However, the project has provided tentative evidence of outcome measures which may be useful for evaluating important aspects of dementia services. Clearly, future development of the measures would need to include evaluation of the data as well as methods of feeding back results to staff to influence care.

The assessment of the practicality, acceptability, and utility of the measures in the evaluation of dementia services may be a unique approach. Project planning seeking maximum ownership of outcome measures by clinicians must ensure that the elements of management support, ${ }^{17}$ benefits of outcome measures, concerns about evaluation, staff involvement in measure selection, and training are considered. This small study highlights the importance of considering the practical issues of outcome measurement, in conjunction with the requirements of the reliability and validity of the outcome measures themselves. The project is a reminder of the need to take into account the practicalities of using outcome measures in routine practice and the importance of taking into account the views of those who will be using the measures on a day to day basis. Facilitating ownership and ensuring acceptability in this way can only improve the likelihood that outcome measures will be used by service providers.

This work is funded by the Mental Health Foundation. We thank all of the staff who were involved in supporting the study, piloting the measures, and providing their invaluable feedback (Harrow and Hillingdon elderly psychiatric service, Parkside (Harrow and Hillingdon elderly psychiatric service, Parkside
elderly psychiatric teams, Riverside elderly mental health team, elderly psychiatric teams, Riverside elderly mental health team, Admiral Nurse Service, East Hertfordshire CPN team and Car-
ers Group, Haymeads Day Hospital), Margaret Butterworth, and Isobel Hunter.

1 Shanks, J. Outcomes in mental health. Outcomes into clinical practice. London: BMJ Publications 1994.

2 Ramsay M, Winget C, Higginson I. Review: measures to determine the outcome of community services for people with dementia. Age Ageing 1995;24:73-83.

3 Baumgarten $M$. The health of persons giving care to the demented elderly: a critical review of the literature. $\mathcal{f}$ Clin Epidemiol 1989;42:1137-48.

4 Ramsay M, Topan C, Higginson I. Outcome of dementia care: views of general practitioners, district nurses, informal carers and specialist teams. Internal report. London: Kensington and Chelsea and Westminster Health Authority, 1994.

5 Ovretveit J. Health service quality. An introduction to quality methods for health services. Oxford: Blackwell Sci Publ, 1992.

6 Alexopoulos GS, Abrams RC, Young RC, Shamoian CA. Cornell scale for depression in dementia. Biol Psychiatry 1988;23:271-84.

7 Vida S, Des-Rosiers P, Carrier L, Gauthier S. Depression in Alzheimer's disease: receiver operating characteristic analysis of the Cornell scale for depression in dementia and the sis of the Cornell scale for depression in dementia and the
Hamilton depression scale. $F$ Geriatr Psychiatry Neurol Hamilton depressi

8 Summers WK, DeBoynton V, Majovski LV. Comparison of seven psychometric instruments used for evaluation of treatment effect in Alzheimer's dementia. Neuroepidemiology 1990;9:193-207.

9 Greene JG, Smith R, Gardiner M, Timbury GC. Measuring behavioural disturbance of elderly demented patients in the community and its effects on relatives: a factor analytic study. Age Ageing, 1992;11:121-6.

10 Cohen S, Kamarck T, Mermelstein R. A global measure of perceived stress. f Health Soc Behav 1983;24:385-96.

11 Hewitt PL, Flett GL, Mosher SW. The perceived stress scale: factor structure and relation to depression symptoms in a psychiatric sample. Fournal of Psychopathology and Behavioral Assessment 1992;14: 247-57.

12 Pbert L, Doerfler LA, Decosimo D. An evaluation of the perceived stress scale in two clinical populations. Fournal of Psychopathology and Behavioral Assessment 1992;14:363-75.

13 Lawton MP, Kleban MH, Moss M, Rovine M, Glicksman A. Measuring caregiver appraisal. $\mathcal{f}$ Gerontol B Psychol Sci Soc Sci 1988;44:61-71.

14 Gilleard CJ, Boyd WD, Watt G. Problems in caring for the elderly mentally infirm at home. Archives of Gerontology and Geriatrics 1982;1:151-8.

15 Gilleard CJ, Gilleard E, Whittick JE. Impact of psychogeriatric day hospital care on the patient's family. Br f Psychiatry 1984;145:487-92.

16 Thomson R, Barton A. Is audit running out of steam? Quality in Health Care 1994;3:225-9.

17 Firth-Cozens J. Building teams for effective audit, Quality in Health Care 1992;1:252-5. 
Appendix: Dementia outcome study: feedback questionnaire

Thank you for taking part in the dementia outcome study. This project is assessing the use of outcome measures in routine practice and we are interested in obtaining staff views. Please complete one of these feedback questionnaires each time you use one of the outcome measures in your routine work.

Please tick the most appropriate response and give comments where indicated.

1. Staff designation:

- Community Psychiatric Nurse

- Senior Psychiatrist

- Social Worker

- Junior Psychiatrist

-Occupational Therapist

-Psychologist

- Nurse

-Other (please specify)

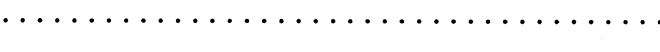

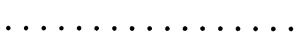

2. Which measure does this feedback relate to?

Patient measures:

- Cornell Scale for Depression in Dementia

-Alzheimer's Deficit Scale

-Behaviour and Mood Disturbance Scale

Carer measures:

-Perceived Stress Scale

- Caregiver Appraisal

- Problem Checklist

3. How long did the measure take to complete?

-5-10 mins

-11-15 mins

$-16-20 \mathrm{mins}$

$-21-30 \mathrm{mins}$

$-30+\operatorname{mins}$

4. Was the measure easy to use on this occasion?

- very easy

- quite easy

- quite difficult

- very difficult

Comments:

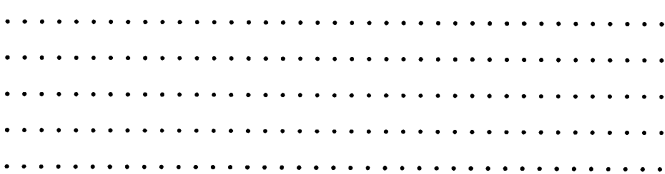

5. Did you feel that the measure provided you with useful information?

- very useful

- quite useful

- not very useful

- not at all useful

Comments:.

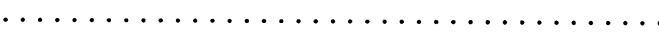

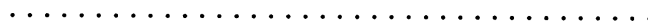

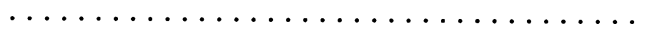

6. How relevant do you feel the measure was to this particular patient/carer?

- very relevant
- quite relevant

- not very relevant

- not at all relevant

Comments:

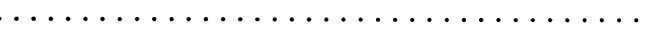

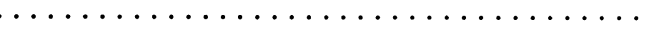

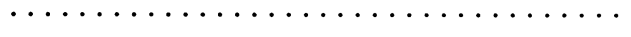

7. Did the carer feel it was relevant to their caregiving situation?

- yes

- no

- unsure

- not applicable

Comments:

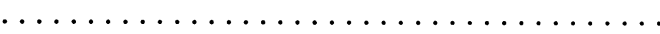

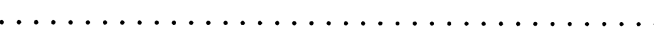

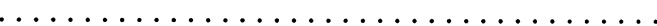

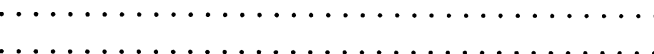

8. Did you or the carer encounter any problems with using the measure (e.g. following the instructions, understanding the items)?

- yes

- no

Please explain:

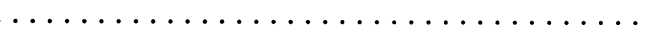

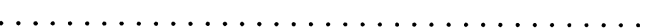

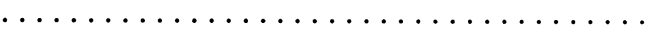

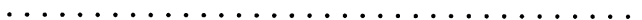

9. Do you think that this measure would be suitable to use in routine practice?

- yes

- no

- unsure

Comments:

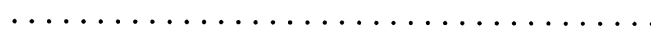

$\ldots \ldots \ldots \ldots \ldots \ldots \ldots \ldots \ldots \ldots \ldots$

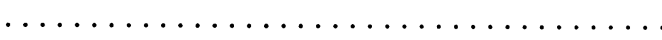

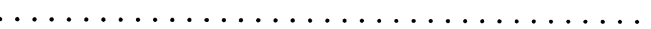

10. How do you think this measure could best be used to facilitate quality of care?

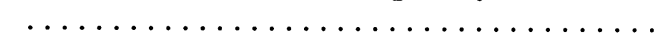

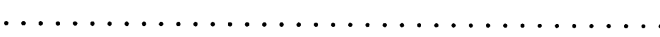

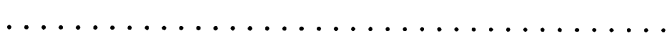

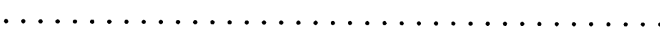

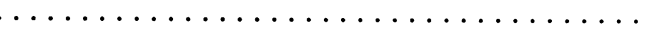

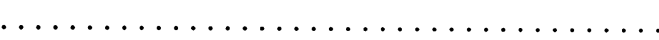

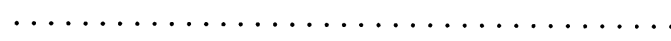

11. Any other comments:

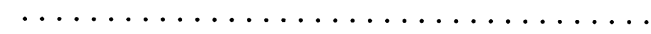

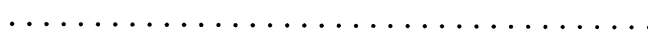

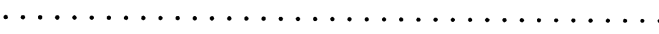

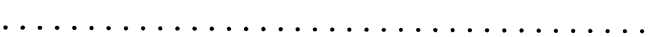

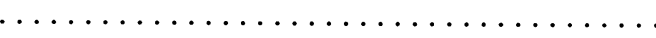

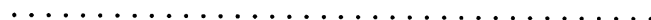

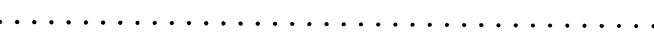

Thank you

Please retain for collection or return to C. Hodgson. The project helpline is open for queries. 